\title{
The use of modified Field's stain in diagnosis of Trichomonas vaginalis
}

\author{
Hamed Abdahhah Hamed ${ }^{1}$, Nashaat Nabil Esmail'2, Marwa Ahmed Aldardiry ${ }^{3}$ and \\ Rashad Mahmoud Mostafa ${ }^{4}$ \\ ${ }^{1}$ Department of Andrology \& S.T.Ds, Faculty of Medicine, Cairo University, Cairo, Egypt \\ ${ }^{2}$ Department of Andrology \& S.T.Ds, Faculty of Medicine, Beni-Seuf, Egypt \\ ${ }^{3}$ Department of Parasitology, Faculty of Medicine, Fayoum University, Egypt \\ ${ }^{4}$ Andrology Unit, Department of Dermatology, Venereology \& Andrology, Faculty of Medicine, \\ Suez Canal University, Egypt
}

Original

Article

\begin{abstract}
Backgroud: Trichomoniasis is not a reportable infection and its control received relatively little emphasis from public health STD control programs although it is the most common curable sexually transmitted disease in young sexually active women.

Purpose: To diagnose trichomoniasis among women attending family planning units and to identify the possible risk factors affecting the disease.

Materials and Methods: The present work was carried on 100 married women attending family planning units in ELFayoum Governorate. Diagnosis of trichomoniasis was done by direct wet mount microscopy, Geimsa staining and modified Field Stain.

Results: The women ages ranged from 19 to 49 years old. The mean women age was $29.7 \pm 7.1$ years old. The majority of women $(62 \%)$ had residence in rural areas and housewives $(82 \%)$. The educational level ranged from illiteracy to high collage. Illiterate women were (14\%). Seventy four women (74\%) had educational level within 8 years of schooling. About $12 \%$ of women had high level education. Only six women $(6 \%)$ had diabetes mellitus. The symptoms reported by the examined women include: $(60 \%)$ increased vaginal discharge and itching, (31\%) genital malodor. While (29\%) women were presented with dyspareunia, (33\%) presented with dysuria and (9\%) with lower abdominal pain. This study demonstrated that Modified Field Stain ( MF) stain, apart from being a rapid stain (20 s) in comparison to Giemsa stain (20 min.), confers sharper staining contrast, which differentiates the nucleus and the cytoplasm of the organism and demonstrated that Vacuoles are more clearly visible in parasites with MF than those stained with Giemsa.

Conclusion: The results of the present study pointed to the benefits of use of modified Field stain in diagnosis of $\mathrm{T}$. vaginalis infection.
\end{abstract}

Key Words: Giemsa stain, modified field stain, trichomonas vaginalis, wet preparation.

Received: 23 July 2017, Accepted: 1 March 2018

Corresponding Author: Rashad Mahmoud Mostafa, Andrology, Sexology \&. STDs, Andrology Unit, Faculty of Medicine, Suez Canal University-Ismailia, Egypt. Tel.: 002-0101393823, E-mail: rashad1010@yahoo.com.

ISSN: 2090-6048, March 2018, Vol.8, No. 1

\section{INTRODUCTION}

Trichomonas vaginalis, the etiologic agent of human trichomoniasis is a protozoan parasite that infects the human urogenital tract ${ }^{[1]}$. Trichomoniasis is the most common curable sexually transmitted disease in young sexually active women. Trichomoniasis spreads through unprotected intercourse with an infected partner ${ }^{[2]}$. Trichomoniasis occurs in $66-100 \%$ of female partners of infected men and $30-80 \%$ of male sexual partners of infected women. The infection may show diversities with respect to socio-cultural properties of the communities changing from a country to another and from a society to another ${ }^{[3]}$. The annual worldwide incidence of trichomoniasis is more than 170 million cases ${ }^{[4]}$. The average incidence in the normal population is approximately $10 \%$ with a range of $0-65 \%$ among different groups. Despite being an easily and readily diagnosed and treated sexually transmitted disease (STD), trichomoniasis is not a reportable infection and its control received relatively little emphasis from public health STD control programs ${ }^{[5]}$. Trichomoniasis is associated with adverse health consequences to women which include: premature rupture of the membranes, preterm delivery of low birth weight infants, adnexitis, 
pyosalpinx, endometritis, and cervical erosion ${ }^{[6]}$. Cervical neoplasia, infertility and a typical pelvic inflammatory disease are additional complications ${ }^{[7]}$. This STD increases the predisposition to human immunodeficiency virus (HIV) sero-conversion ${ }^{[8]}$. Therefore, Different methods are used for the laboratory diagnosis of trichomoniasis such as wet mount, Giemsa staining methods, culture, latex agglutination, enzyme linked immune-sorbent assay and molecular techniques as polymerase chain reaction $(\mathrm{PCR})^{[9]}$. Stained smear techniques because cultivation methods are relatively slow and wet mount preparation lacks sensitivity, the staining of parasites in fixed and unfixed smears is introduced ${ }^{[10]}$. Different staining techniques include: Gram, Giemsa, Wright's, acridine orange, Leishman, periodic acid-schiff, Fontana stain and Papanicolaou (Pap) smear are preferred and used to improve the sensitivity of direct microscopy ${ }^{[11]}$. Giemsa Staining demonstrates the morphological characteristics of $\mathrm{T}$. vaginalis .There are four free anterior flagella and the fifth posterior flagellum. The undulating membrane extends to about half way across the organism. A slender hyaline, rodlike structure, called an axostyle commences at the nucleus and bisects the protozoan longitudinally. It protrudes through the posterior of the parasite terminating in a sharp point. A prominent nucleus in T. vaginalis is located in its anterior portion $^{[12]}$. Modified Field's Stain (MF) staining, apart from being a rapid stain (20 s), confers sharper staining contrast, which differentiates the nucleus and the cytoplasm of the organism when compared to Giemsa and Gram staining especially on parasites from spiked urine samples. The alternative staining procedure offers in a diagnostic setting a rapid stain that can easily visualize the parasite with sharp contrasting characteristics between organelles especially the nucleus and cytoplasm. Vacuoles are more clearly visible in parasites stained with MF than when stained with Giemsa. Modified Field's Stain (MF) staining does not only record the morphological changes and retain internal structural details but also provides a reliable and rapid viability assay for the parasite ${ }^{[13]}$.

\section{AIM OF STUDY}

To diagnose trichomoniasis among women attending family planning units and to identify the possible risk factors affecting the disease.

\section{PATIENTS AND METHODS}

This study was a retrospective, descriptive study, conducted on 100 women visiting the family planning clinics at primary health care units of EL-Fayoum Governorate - Egypt for routine checkup between June and July, 2013. These clinics were selected because of high utilization rate ( $>50$ women/month). In addition, the selected clinics represent both rural and urban sectors of the community. At the same time, they are the nearest units to the Parasitology laboratory in the Faculty of Medicine, Fayoum University for quick examination of the collected specimens. The study sample will randomly select irrespective to complains, socio-economic standards and medical history (even diabetic patient). The data were collected using a structured questionnaire interview. It is focused on:

1- Socio-demographic characteristics in terms of women's age, address, residence area (rural or urban), educational level, occupation and hygienic practices,

2- History of certain diseases especially: diabetes mellitus and

3- Signs and symptoms of vaginitis in the form of increased vaginal discharge, its color, odor, consistency and any associated cervical abnormalities. The presence of associated symptoms such as itching, burning, lower abdominal pain and painful intercourse.

The purpose of this study was explained to each woman before testing. Serial number will be used. Each woman was given the same serial number on the filled questionnaire. All examined women were subjected to: a) - Detailed general physical examinations, b) Systemic and pelvic examinations, c) Examination per speculum: to look for vaginal discharge, to evaluate the condition of the vagina and cervix and to collect vaginal discharge. Vaginal discharge specimens were collected from the posterior fornix and mucosa of the vagina by a sterile cotton wool swab sticks. Each sample was labeled using serial number. For each woman samples was collected as follow: The swab was kept in small Eppendorf tube containing approximately $0.5 \mathrm{ml}$ of warm normal saline solution $(0.9 \% \mathrm{NaCl})$ for saline wet mount microscopy, Giemsa staining and modified MF staining. The swab swirls gently in the saline to make a heavy suspension. Ream the swab on the side of the Eppendorf tube to expel fluid. Discard the swab in appropriate container. The collected samples were quickly transported to the Parasitology laboratory for complete testing and incubation. Each sample was subjected to the following: 1. Saline wet mount microscopy, 2. Giemsa staining, and 3. MF staining.

1- SALINE WET MOUNT MICROSCOPY: Saline wet mount microscopy was done according to Garcia $^{[14]}$ : Reagents: Normal sterile saline $(0.9 \%) \square$ Modified D'Antoni's Stock Iodine prepared according to Garcia (1999): $\square$ Ingredients: $\square$ Potassium iodide (ELNasr.CO., Egypt)..1.0 g Powdered iodine crystals (ELNasr.CO., Egypt)..1.5 g Distilled water..100ml Preparation of D'Antoni's iodine stock: 1. Dissolve the previous ingredients in an appropriate glass flask, 2. The D'Antoni's iodine solution is saturated with iodine, with some excess crystals left in the bottle. The iodine solution is stored in a brown bottle at room temperature. The stock solution remains good as long as an excess of iodine crystals remains at the bottom of the bottle, 3. Label the dark bottle as D'Antoni's stock iodine with the preparation date and 
an expiration date of 1 year and 4. Aliquot small amounts of stock iodine solution into brown dropper bottles for routine, daily use. The expiration date will be 30 to 60 days, depending on the amount of fading of the solution from the normal strong-tea color. The use of a brown bottle will lengthen the expiration date.

Procedure of wet mount microscopy: Apply a drop of the collected sample in warm normal saline solution to a small area of clean grease-free microscopic slide. Cover the specimen with a cover slip. Be careful not to trap air bubbles under the cover-slip. Read the slide immediately. Examine the entire smear under the microscope for motile T. vaginalis with low power objective (x10). Suspicious objects examined with high power objective (x40) (15). If motile flagellates are seen, The trophozoites of $\mathrm{T}$. vaginalis are present. If non-motile organisms are visible after staining with D'Antoni's iodine, trophozoites of T. vaginalis are present. The organisms do not have to be quantitated. IF no flagellated organisms are seen, report the specimen as negative for $\mathrm{T}$. vaginalis. Test is dependent on demonstrating the motility of $\mathrm{T}$. vaginalis that loses their motility rapidly after leaving the vaginal environment. All specimens should be held at room temperature because refrigerator temperature will inhibit motility and have deleterious effect on the organisms. Wet mount examination should be performed within one hour of collection for optimal results.

GIEMSA STAINING Reagents: 1. Giemsa Stain: Concentrated liquid stock is available commercially prepared by Egyptian Dignost. Co., Egypt. 2. Absolute Methanol: acetone-free (El-Gomhoria. Co., Egypt). 3. Phosphate Buffer Solution pH 7.2 (Giemsa buffer): The stain must be diluted for use with phosphate buffer solution $\mathrm{pH}$ 7.2.

Phosphate Buffer Solution (Giemsa buffer): Prepare a $67 \mathrm{mmol} / \mathrm{L}$ phosphate buffer, $\mathrm{pH} \mathrm{7.2,} \mathrm{according} \mathrm{to}$ Hawkey and Lewis (16) as follow: 1. Solution: A: prepare a $67 \mathrm{mmol} / \mathrm{L} \mathrm{Na} 2 \mathrm{HPO} 4$ by adding 9.5 grams of disodium hydrogen phosphate (anhydrous salt) (Oxford Lab., India) to 1 liter of distilled water, 2. Solution B: prepare a 67 mmol / L NaH2PO4. H2O by dissolving 9.2 grams of sodium dihydrogen phosphate (Oxford Lab., India) to 1 liter of distilled water. Mix $72 \mathrm{ml}$ of solution A with 28 $\mathrm{ml}$ of solution B and $900 \mathrm{ml}$ of distilled water Working Solution: Stock Giemsa solution. 1 part. Phosphate buffer (pH 7.2) 20 parts.

Staining Procedure: The procedure was done according to Radonjic et al. ${ }^{[17]}$ : 1 . Apply the specimen to a small area of a clean microscope slide. 2. Allow air to dry the smear, 3. Fix in absolute methanol for 1 minute (in slide- staining rack) and dry again, 4. Place the slide in Giemsa solution (freshly prepared) and stain for desired time depending on the stain dilution used (20 minutes at 1: 20 dilution), 5. Rinse the slide with phosphate buffer to remove excess stain solution, 6. Drain the slide in vertical position and allow to air dry, (don't apply a cover slip) and 7. Examine the entire smear under the microscope by $(\mathrm{x} 100)$ objective lens to detect violet pear shaped trophozoites with characteristic features.

Modified Field's' Stain (MF) staining Reagents: 1. $6.25 \mathrm{~g}$ Field's' stain $2.1 \mathrm{gm}$ eosin yellow in $500 \mathrm{ml}$ of pure methanol (0.2) Technique of preparation: Field's' stain A: Mix Field's' stain A in $500 \mathrm{ml}$ of hot water ,shake thoroughly, after mixing properly, filter with filter paper and pour into a clean bottle. Field's' stain B (modified): mix 0.2 eosin in pure methanol, shake thoroughly, after mixing properly, filter with filter paper and pour into a clean bottle, ready to use. Staining Procedure: 1. smear of sediment containing $\mathrm{T}$. vaginalis were dried at room temperature.

\section{RESULTS}

In the present study, 100 married females from different rural and urban areas in EL-Fayoum Governorate Egypt were examined for $\mathrm{T}$. vaginalis infection. The study represents a randomly selected sample of women visiting the family planning clinics (FPCs) at primary health care units. They were coming for routine checkup between January and March, 2014. They were visiting the family planning units for new contraceptive methods. All participants in this study had regular marital life.

In the present study, the mean age of the studied group was $(29.7 \pm 7.1)$ years old, and the mean marriage duration was $(8.6 \pm 6.3)$ years. The frequency of medical history of diabetes was $6 \%$ among the studied group, and frequency of different clinical symptoms in $60 \%$ of women had increase in vaginal discharge, and vaginal itching, followed by $33 \%$ had dysurea, $31 \%$ had vaginal malodor, $29 \%$ had dyspareunia and finally only $9 \%$ had lower abdominal pain.

The frequency of clinical examination findings among the studied group illustrated that $36 \%$ of women had pallor in general examination, $11 \%$ had abnormal abdominal and bimanual examination, all women had normal external pelvic examination, $44 \%$ of women were using intrauterine device, and only $9 \%$ of them had abnormal cervical examination as shown in Table (1). 
Table (1): Description of clinical examination among study group

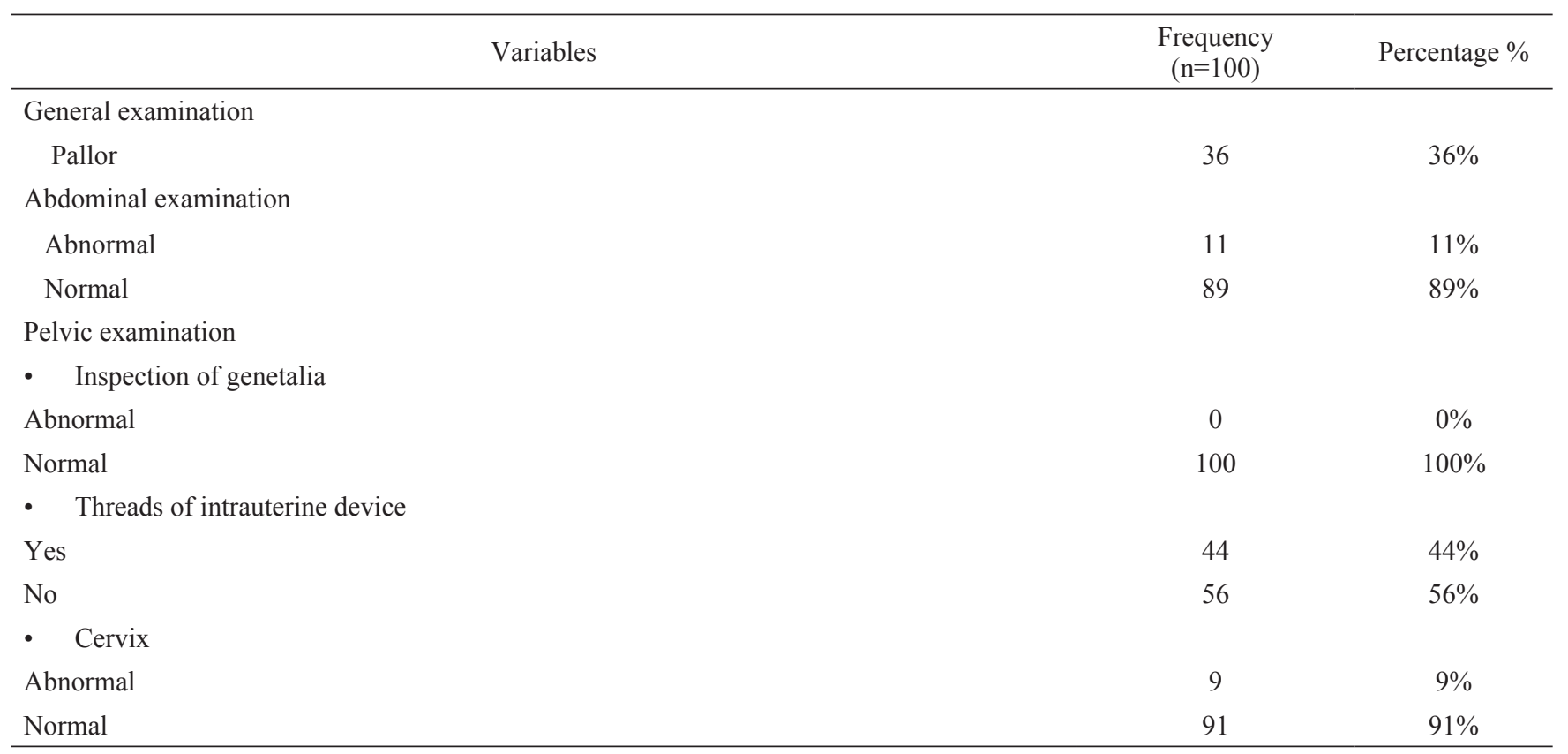

This study showed vaginal discharge different characters as it was fishy odor in $4 \%$ of the women, white discharge in 53\% of the women and watery vaginal discharge in $81 \%$ of the women, as shown in table (2).

Table (2): Description of vaginal discharge characters among study group.

\begin{tabular}{ccc}
\hline Variables & $\begin{array}{c}\text { Frequency } \\
(\mathrm{n}=100)\end{array}$ & Percentage \% \\
\hline
\end{tabular}

Odor

- Fish odor

4

$4 \%$

Color

- White

53

$53 \%$

- Yellow

38

$38 \%$

- Gray

9

$9 \%$

Consistency

- Watery

81

13

$13 \%$

- Thick creamy

6

$6 \%$
In the present study, Frequency of different diagnostic tests outcomes in $24 \%$ of women included in the study were positive T.vaginalis by, Field staining tests and Giemsa staining; on the other hand only $15 \%$ of them were positive by Fresh saline wet mount microscopy test as shown in table (3).

Table (3): Description of different laboratory tests among study group.

\begin{tabular}{ccc}
\hline Variables & $\begin{array}{c}\text { Frequency } \\
(\mathrm{n}=100)\end{array}$ & Percentage \% \\
\hline
\end{tabular}

Fresh saline wet mount microscopy

Negative

85

$85 \%$

Positive

24

$24 \%$

Giemsa staining

Negative

38

$38 \%$

Positive

9

$9 \%$

Field staining

\begin{tabular}{lll} 
Negative & 76 & $76 \%$ \\
Positive & 24 & $24 \%$ \\
\hline
\end{tabular}


Table (4) illustrated that there is no statistically significance difference between different Field staining tests outcomes (positive and negative) ( $p$-value $>0.05)$ as regards to all demographic characters (residence, marital state, education level, and occupation). Also, there was no statistically significant difference between different Field staining tests outcomes (positive, and negative) ( $p$-value $>0.05)$ as regards to both age and marital duration.

Table (4): Comparison of demographic characters among different filed staining outcomes.

\begin{tabular}{|c|c|c|c|c|c|c|}
\hline \multirow[t]{2}{*}{ Variables } & \multicolumn{2}{|c|}{$\begin{array}{l}\text { Negative MF } \\
(n=76)\end{array}$} & \multicolumn{2}{|c|}{$\begin{array}{l}\text { Positive MF } \\
(n=24)\end{array}$} & \multirow[t]{2}{*}{ p-value } & \multirow[t]{2}{*}{ Sig. } \\
\hline & No. & $\%$ & No. & $\%$ & & \\
\hline \multicolumn{7}{|l|}{ Residence } \\
\hline Rural & 45 & $59.2 \%$ & 17 & $70.8 \%$ & \multirow{2}{*}{0.3} & \multirow{2}{*}{ NS } \\
\hline Urban & 31 & $40.8 \%$ & 7 & $29.2 \%$ & & \\
\hline \multicolumn{7}{|l|}{ Educational level } \\
\hline Elementary/ intermediate & 57 & $75 \%$ & 17 & $70.8 \%$ & \multirow{3}{*}{0.7} & \multirow{3}{*}{ NS } \\
\hline Illiterate & 11 & $14.5 \%$ & 3 & $12.5 \%$ & & \\
\hline Secondary /university & 8 & $10.5 \%$ & 4 & $16.7 \%$ & & \\
\hline \multicolumn{7}{|l|}{ Occupation } \\
\hline House wife & 62 & $81.6 \%$ & 20 & $83.3 \%$ & \multirow[b]{2}{*}{0.9} & \multirow[b]{2}{*}{ NS } \\
\hline Employed & 14 & $18.4 \%$ & 4 & $16.7 \%$ & & \\
\hline
\end{tabular}

Table (5) illustrated that there is statistically significant difference between different Field staining tests outcomes (positive, and negative) ( $p$-value $<0.05)$ regarding to increase discharge, and vaginal malodor with high percentage among positive MF test, on the other hand there is no statistically significance difference with $p$-value $>0.05$ as regards to medical history of diabetes and other clinical symptoms.
In the present study, there is was no statistically significant difference between different Field staining tests outcomes (positive and negative) ( $p$-value $>0.05$ ) concerning general, abdominal and pelvic examination signs as shown in table 6

Table (5): Comparison of medical history and clinical symptoms among different filed staining outcomes.

\begin{tabular}{|c|c|c|c|c|c|c|}
\hline \multirow[t]{2}{*}{ Variables } & \multicolumn{2}{|c|}{$\begin{array}{l}\text { Negative MF } \\
\quad(\mathrm{n}=76)\end{array}$} & \multicolumn{2}{|c|}{$\begin{array}{l}\text { Positive MF } \\
\quad(\mathrm{n}=24)\end{array}$} & \multirow[t]{2}{*}{ p-value } & \multirow[t]{2}{*}{ Sig. } \\
\hline & No. & $\%$ & No. & $\%$ & & \\
\hline \multicolumn{7}{|l|}{ Medical history } \\
\hline Not-diabetic & 72 & $94.7 \%$ & 22 & $91.7 \%$ & \multirow[b]{2}{*}{0.6} & \multirow[b]{2}{*}{ NS } \\
\hline Diabetic & 4 & $5.3 \%$ & 2 & $8.3 \%$ & & \\
\hline \multicolumn{7}{|l|}{ Clinical symptoms } \\
\hline Increase discharge & 39 & $51.3 \%$ & 21 & $87.5 \%$ & 0.002 & HS \\
\hline Vaginal malodor & 17 & $22.4 \%$ & 14 & $58.3 \%$ & 0.002 & HS \\
\hline Vaginal itching & 47 & $61.8 \%$ & 13 & $54.2 \%$ & 0.6 & NS \\
\hline Dyspareunia & 21 & $27.6 \%$ & 8 & $33.3 \%$ & 0.6 & NS \\
\hline Dysurea & 25 & $32.9 \%$ & 8 & $33.3 \%$ & 0.9 & NS \\
\hline Lower abdominal pain & 7 & $9.2 \%$ & 2 & $8.3 \%$ & 0.9 & NS \\
\hline
\end{tabular}


Table (6): Comparison of clinical examination among different filed's staining outcomes.

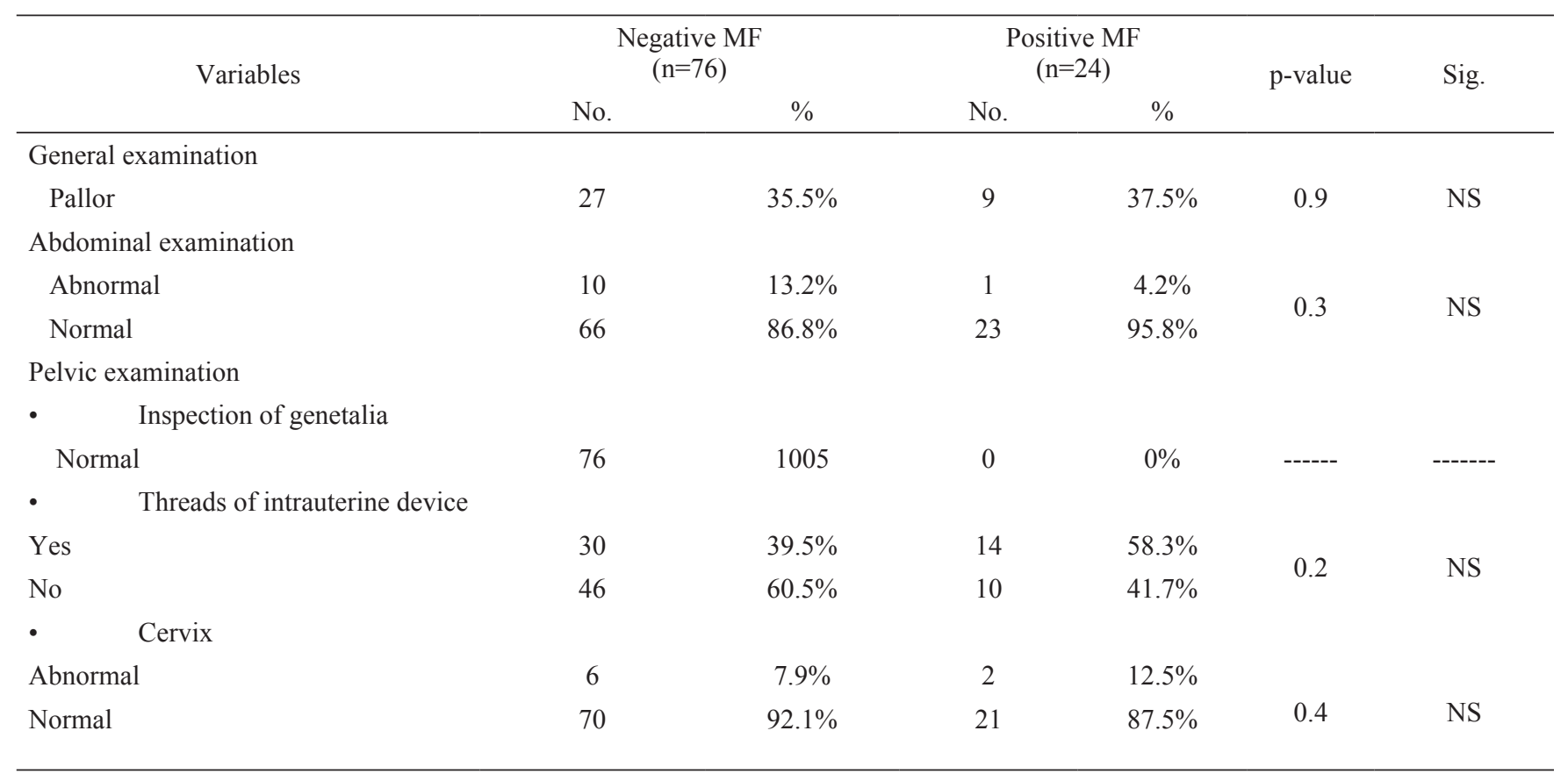

In the present study, that there was a statistically significant difference between different Field staining tests outcomes (positive, and negative) ( $p$-value $<0.05$ ) as regards to fish odor and consistency of vaginal discharge with high percentage of fish odor and watery discharge among positive MF test, on the other hand there is no statistically significance difference with $p$-value $>0.05$ as regards to color of vaginal discharge as shown in figure as shown in table (7).

Table (7): Comparison of vaginal discharge characters among different filed staining outcomes.

\begin{tabular}{|c|c|c|c|c|c|c|c|}
\hline & \multirow[t]{2}{*}{ Vaginal discharge } & \multicolumn{2}{|c|}{$\begin{array}{l}\text { Negative MF } \\
\quad(n=76)\end{array}$} & \multicolumn{2}{|c|}{$\begin{array}{l}\text { Positive MF } \\
\quad(n=24)\end{array}$} & \multirow[t]{2}{*}{$\mathrm{p}$-value } & \multirow[t]{2}{*}{ Sig } \\
\hline & & No. & $\%$ & No. & $\%$ & & \\
\hline \multicolumn{8}{|c|}{ Odor } \\
\hline • & Fish odor & 1 & $1.3 \%$ & 3 & $12.5 \%$ & 0.04 & $\mathrm{~S}$ \\
\hline \multicolumn{8}{|c|}{ Color } \\
\hline - & White & 43 & 56.6 & 10 & $41.7 \%$ & & \\
\hline - & Yellow & 27 & $35.5 \%$ & 11 & $45.8 \%$ & 0.4 & NS \\
\hline - & Gray & 6 & $7.9 \%$ & 3 & $12.5 \%$ & & \\
\hline \multicolumn{8}{|c|}{ Consistency } \\
\hline - & Thick creamy & 12 & $15.8 \%$ & 1 & $4.2 \%$ & & \\
\hline - & Watery & 62 & $81.6 \%$ & 19 & $79.2 \%$ & 0.02 & $\mathrm{~S}$ \\
\hline • & Frothy & 2 & $2.6 \%$ & 4 & $16.7 \%$ & & \\
\hline
\end{tabular}


In this study, the Sensitivity and specificity test of Fresh mount and Giemsa test in comparison with Filed saline test in diagnosis of T.vaginalis illustrated that fresh saline wet mount test probability of being true positive was $(81.3 \%)$, more than being false positive when repeat test 100 times with sensitivity
(62.5\%), positive predictive (100\%), and specificity (100\%), negative predictive (89.4\%), and for Giemsa staining sensitivity $(100 \%)$, positive predictive $(100 \%)$, and specificity $(100 \%)$, negative predictive $(100 \%)$ as shown in table (8).

Table (8): Sensitivity and specificity of Fresh mount and Giemsa test in comparison with Field saline test in diagnosis of T.vaginalis.

\begin{tabular}{lcccc}
\hline Variable & Sensitivity & Specificity & +ve predictive & -ve predictive \\
\hline $\begin{array}{l}\text { Fresh saline wet } \\
\text { mount microscopy }\end{array}$ & $62.5 \%$ & $100 \%$ & $100 \%$ & $89.4 \%$ \\
Giemsa staining & $100 \%$ & $100 \%$ & $100 \%$ & $100 \%$ \\
\hline
\end{tabular}

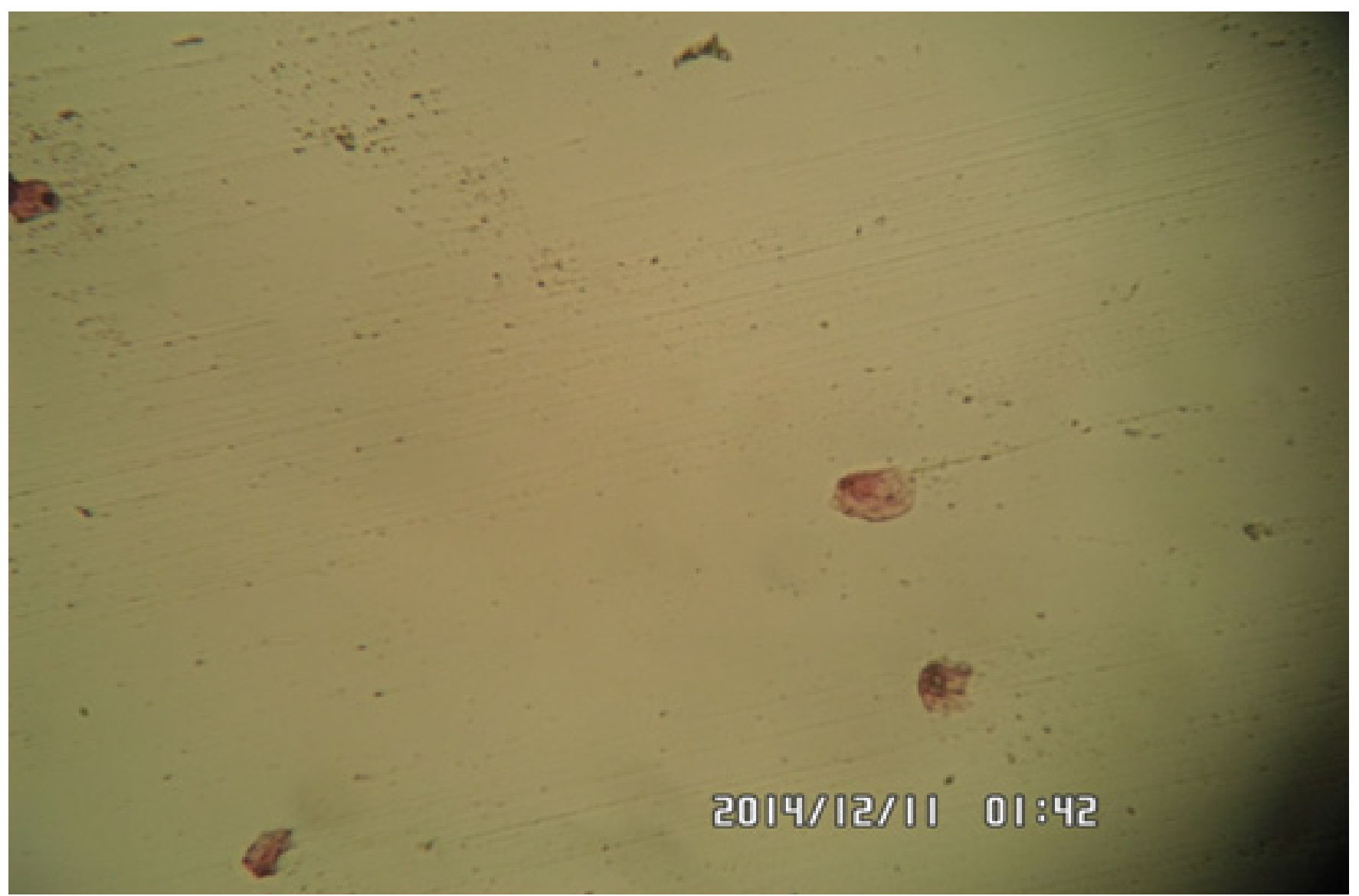

Fig. 1: Giemsa staind T.Vaginalis (at magnification $100 \mathrm{X}$ ). 


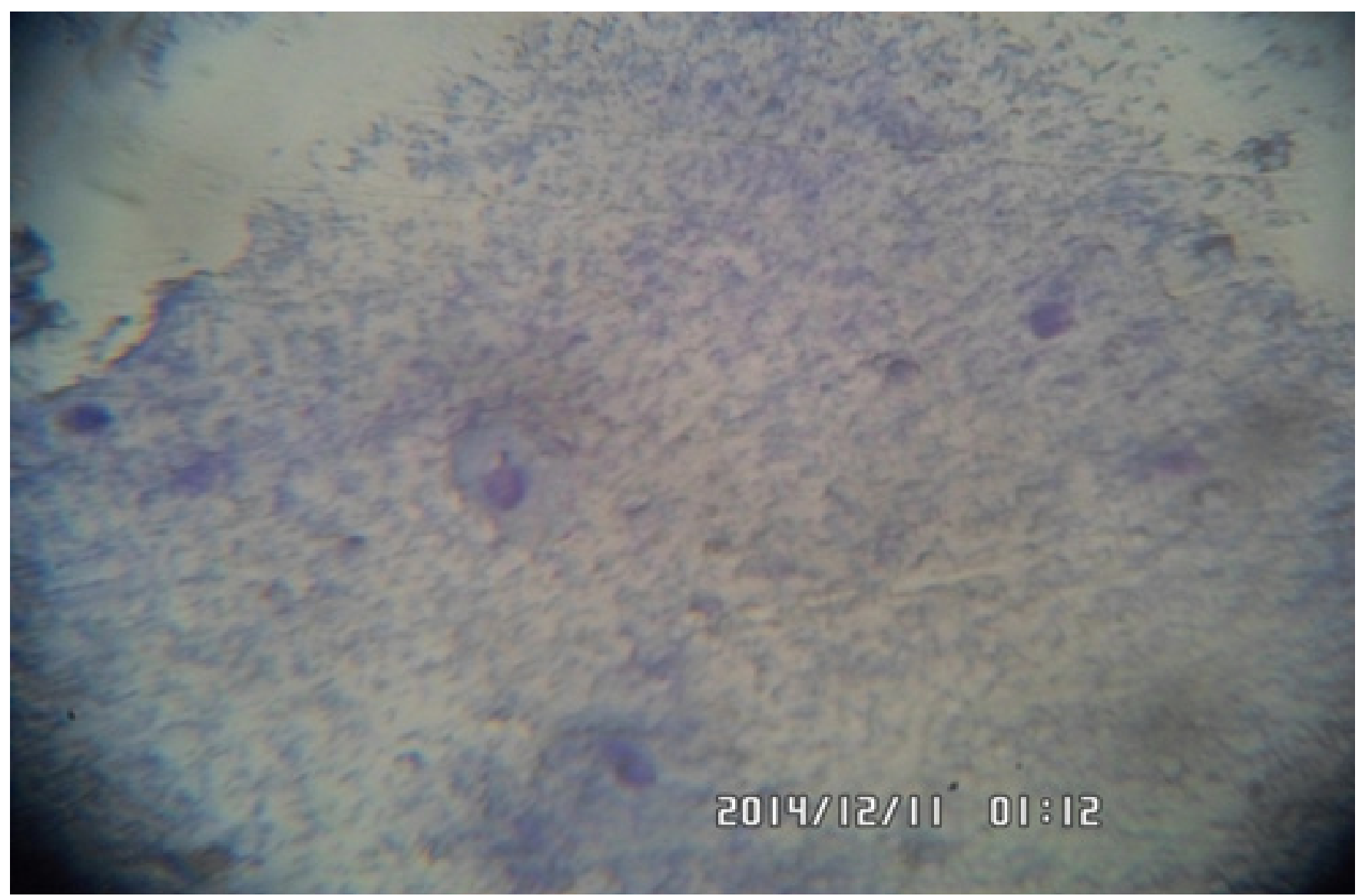

Fig. 2: Modified Field stain stained T.Vaginalis. (at magnification $100 \mathrm{X}$ ).

\section{DISCUSSION}

Trichomonas vaginalis, the etiologic agent of human trichomoniasis is a protozoan parasite that infects the human urogenital tract ${ }^{[1]}$. Trichomoniasis is the most common curable sexually transmitted disease in young sexually active women. Trichomoniasis spreads through unprotected intercourse with an infected partner ${ }^{[2]}$. The present study reported the highest percent of positive $\mathrm{T}$. vaginalis infections $(68 \%)$ observed among females with age below thirty years old. However, only thirty women $(32 \%)$ with age above thirty years old had positive $\mathrm{T}$. vaginalis infections. This in agreement with Mason et al., who reported increased positive cases of $\mathrm{T}$. vaginalis with age below 30 years. They found strongest statistical association at this young age that certainly reflecting early sexual activity ${ }^{[18]}$.

This study also showed that, the majority of women 62 $(62 \%)$ had residence in rural areas while thirty eight women (38\%) lived in urban areas. The association between area of residence of females included in the study and the risk of $\mathrm{T}$. vaginalis infection was evaluated in the current work. The study also showed the highest percent $(70.8 \%)$ of infection with $\mathrm{T}$. vaginalis was detected among females lived in rural areas followed by females lived in urban areas $(29.2 \%)$. In accordance, Fotinatos et al., detected significant association between $\mathrm{T}$. vaginalis infection and area of residence. The infection with $\mathrm{T}$. vaginalis among females lived in rural areas was higher than those lived in urban areas. The authors explained their results by fewer health care services, minimal or no women's health awareness programs and transport difficulties to medical facilities $^{[19]}$.

The present work studied the relation between clinical data (symptoms and signs) and trichomoniasis. The commonest complains among $\mathrm{T}$. vaginalis positive females were increased vaginal discharge $(87.5 \%)$, malodor $(58.3 \%)$, vaginal itching $(54.2 \%)$, dysuria $(33.3 \%)$, dyspareunia $(33.3 \%)$ and lower abdominal pain $(8.3 \%)$. There was significant association between trichomoniasis and the presence of malodor and discharge $(p=<0.05)$. Similar reports found by Lawing et al. ${ }^{[20]}$ and Swygard et al..$^{[21]}$ who reported seventy-five percent $(57 \%)$ of women with trichomoniasis complained of vaginal symptoms such as discharge, malodour, and itching. The presence of these symptoms was not significantly associated with a positive T. vaginalis infections $(P=0.47)$.

Per-speculum examination revealed that out of nine women presented with red spotting on the cervix, only two women were diagnosed positive for $\mathrm{T}$. vaginalis infection. This finding showed no statistically significant association 
with trichomoniasis $(P=0.4)$. Swygard et al. reported that the presence of abnormal discharge color and the character of the vaginal discharges were more significantly associated with $T$. vaginalis infection in positive cases, While cervical abnormalities were not significantly associated with $\mathrm{T}$. vaginalis infection $^{[22]}$.

In the present study, wet mount, Giemsa staining and MF stain were performed for all vaginal discharge samples taken from women attending FPCs. Examination of vaginal discharge samples revealed $\mathrm{T}$. vaginalis trophozoites. Regarding the shape and size of $\mathrm{T}$. vaginalis trophozoites, similar findings were reported by Tasca and De-Carli, who found that urogenital trichomonads vary in size and shape. Physicochemical conditions $(\mathrm{pH}$, temperature and oxygen tension) affect the shape of trichomonads. The axenic cultures (liquid media) for example: trypticase-yeast extract-maltose (TYM) with or without low concentrations of agar and without solid food particles, the organisms are usually ellipsoidal, ovoid or spheroidal in shape ${ }^{[23]}$.

In the present study $\mathrm{T}$. vaginalis infections were detected in vaginal swabs by different methods. Out of 100 women, fifteen cases were diagnosed by wet mount microscopy $(15 \%)$. 24 women $(24 \%)$ were detected by Giemsa and MF stain. The low sensitivity of wet mount (15\%) found in the current work could be explained by that wet preparation sensitivity is highly dependent on the expertise of the microscopist, prompt transport and laboratory processing before the organism lyses or loses motility. Schwebke et al., assumed that wet preparation is the most often used diagnostic test for trichomoniasis in women. It is inexpensive and relatively easy to interpret and provides immediate results to facilitate patient treatment. However, the wet preparation has limited sensitivity compared to culture $^{[24]}$.

The reported results for wet mount test in the current work remain within range as confirmed by (Kaydos-Daniels ) who reported that the sensitivity of wet mount compared to culture was 12 to $91 \%{ }^{[25]}$. Jatatu et al., reported low sensitivity (35.69\%) of wet mount preparation with respect to culture medium ${ }^{[26]}$. Abd El-Aal et al., compared the results of wet mount with the "gold standard" culture (on modified diamond) results. The sensitivity of wet mount for the presence of $\mathrm{T}$. vaginalis was low (only $33.3 \%)^{[27]}$.

Regarding the reported results of Giemsa staining in the current work remain within range as confirmed by Malla et al., who noted that the sensitivity of the stained slides ranges between 30 and $85 \%$ when compared with culture methods ${ }^{[28]}$.

The current study demonstrated that Modified Field Stain (MF) stain, apart from being a rapid stain (20 s) in comparison to Giemsa stain (20 min.), confers sharper staining contrast, which differentiates the nucleus and the cytoplasm of the organism and demonstrated that Vacuoles are more clearly visible in parasites stained with MF than those stained with Giemsa. This was in accordance with Afzan et al., who that stated that Modified Field Stain (MF) staining does not only record permanently the morphological changes and retain internal structural details but also provides a reliable and rapid viability assay for the parasite.

\section{CONCLUSION}

The results of the present study pointed to the benefits of use of modified Field stain in diagnosis of $\mathrm{T}$. vaginalis infection. Also, the advantage of being a rapid stain $(20 \mathrm{~s})$ in comparison to Giemsa stain $(20 \mathrm{~min}$.) and confers sharper staining contrast, which differentiates the nucleus and the cytoplasm of the organism and demonstrated that Vacuoles are more clearly visible in parasites stained with MF than those stained with Giemsa. The results indicated that the rate of infection was elevated among house-wives and educated females lived in rural areas in EL-Fayoum Governorate. The results also proved that using modified Field stain and Giemsa stain gave higher detection rate of T. vaginalis than wet mount. From the above mentioned results, the health care providers and females irrespective to their standards should be aware of this infection. Further studies should be done to assess use of modified Field stain in comparison to culture on Diamond medium and PCR in diagnosis of Trichomoniasis. Also, combination of direct wet mount, Giemsa staining and culture of vaginal swabs are recommended in correct laboratory diagnosis of this parasite. So, treatment and control of infection becomes easy.

\section{CONFLICT OF INTERST}

There are no conflict of interests.

\section{REFERENCES}

1. Kaul, P.; Gupta, I.; Sehgal, R. and Malla, N. (2004): Trichomonas vaginalis: random amplified polymorphic DNA analysis of isolates from symptomatic and asymptomatic women in India. Parasitol. Int., 53: 255-262.

2. Fotinatos, N.; Warmington, A.; Walker, T. and Pilbeam, M. (2008): Trichomonas vaginalis in Vanuatu. Aust. J. Rural Health. 16(1): 23-27

3. Yazar, S.; Dağc1, H.; Aksoy, Ü.; Üstün, Ş.; Akısu, Ç.; Ak, M. and Daldal, N. (2002): Frequency of Trichomonas vaginalis among women having vaginal discharge, in Izmir, Turkey. İnönü. Üniv. T1p. Fak. Derg., 9(3):159-161. 
4. World Health Organization (2004): Global prevalence and incidence of selected curable sexually transmitted infections: overview and estimates. World Health Organization, Geneva, Switzerland.

5. Verteramo, R.; Calzolari, E.; Degener, M.; Masciangelo, R. and Patella, A. (2008): Trichomonas vaginalis infection: Risk indicators among women attending for routine gynecologic examination. J. Obstet. Gynaecol. Res., 34(2): 233-237.

6. Petrin, D.; Delgaty, K.; Bhatt, R. and Garber, G. (1998): Clinical and microbiological aspects of Trichomonas vaginalis. Clin. Microbiol. Rev., 11(2): 300-317.

7. Moodley, P.; Wilkinson, D.; Connolly, C.; Moodley, J. and Sturm, A. (2002): Trichomonas vaginalis is associated with pelvic inflammatory disease in women infected with human immunodeficiency virus. Clin. Infect. Dis., 34(4):519-522.

8. Guenthner, P.; Secor, W. and Dezzutti, C. (2005): Trichomonas vaginalis induced epithelial monolayer disruption and human immunodeficiency virus type-1(HIV-1)replication: implications for the sexual transmission of HIV-1. Infect. and Immun. 73(7):4155-4160.

9. Radonjic, V.; Dzamic, M.; Mitrovic, M.; Arsic Arsenijevic S.; Popadic, M.; Kranjcic Zec, F. (2006): Diagnosis of Trichomonas vaginalis infection: The sensitivities and specificities of microscopy, culture and PCR assay. Eur. J. Obstet. Gynecol. Reprod. Biol., 126(1): 116-120.

10. Petrin, D.; Delgaty, K.; Bhatt, R. and Garber, G. (1998): Clinical and microbiological aspects of Trichomonas vaginalis. Clin. Microbiol. Rev., 11(2): 300-317.

11. Aslan, D. L.; McKeon, D. M.; Stelow, E. B.; Gulbahce, H. E.; Kjeldahl, K. and Pambuccian, S. E. (2005): The diagnosis of Trichomonas vaginalis in liquid-based Pap tests: Diagn Cytopathol. 2005 May; 32(5):253-9

12. Petmitr, C. P.; Ramdja, M. and Kajorndechakiat, S. (2000): In vitro sensitivity of Trichomonas vaginalis to DNA Topoisomerase II inhibitors. South East Asian J. Trop. Med. Public health. 31(1):118-122.

13. Afzan,M.Y.;Sivanandan,S.;Kumar,G.S.(2010): Modified Field stain-a rapid stain for Trichomonas Vainalis .Diagn. microbio. and infect. Dis. (68) :159-162.
14. Garcia, S.L. (1999): Methods for urogenital tract specimens. In: practical guide to diagnostic Parasitology. Washington, D.C., USA: A.S.M. Press. 134-139.

15. Marrazzo, J.; Ocbamichael, N.; Meegan, A. and Stamm, E. (2007): Laboratory diagnosis of sexually transmitted diseases. In: the practitioner's handbook for the management of STDs, 4th Edition. Washington, USA: Seattle. 70-91.

16. Hawkey, M. P. and Lewis, A. D. (2004): Medical Bacteriology: a practical approach, illustrated 2nd edition. USA: Oxf. Uni. Press.374.

17. Radonjic, V.; Dzamic, M.; Mitrovic, M.; Arsic Arsenijevic S.; Popadic, M.; Kranjcic Zec, F. (2006): Diagnosis of Trichomonas vaginalis infection: The sensitivities and specificities of microscopy, culture and PCR assay. Eur. J. Obstet. Gynecol. Reprod. Biol., 126(1): 116-120.

18. Mason,P.R.; Fiori,P.L.; Cappuccinelli,P.; Rappelli, P. and Gregson, S. (2005): Seroepidemiology of Trichomonas vaginalis in rural women in Zimbabwe and patterns of association with HIV infection. Epidemiol. Infect., 133(2): 315-338.

19. Fotinatos, N.; Warmington, A.; Walker, T. and Pilbeam, M. (2008): Trichomonas vaginalis in Vanuatu. Aust. J. Rural Health. 16(1): 23-27

20. Thompson, D. A.; Tsai, Y. K.; Gilman, R. H.; Vivar, A. and Calderon, M. (2000): Sexually transmitted diseases in a family lanning and an antenatal clinic in Peru: limitations of current practices and analysis of the use of potential markers, $\mathrm{pH}$ testing and Whiff testing. Sex. Transm. Dis., 27(7): 386-392.

21. Lawing, L. F.; Hedges, S. R. and Schwebke, J. R. (2000): Detection of trichomonosis in vaginal and urine specimens from women by culture and PCR. J. Clin. Microbiol., 38(10): 3585-3588.

22. Swygard, H.; Sena, A. C.; Hobbs, M. M. and Cohen, S. M. (2004): Trichomoniasis: clinical manifestations, diagnosis and management. Sex. Transm. Infect., 80:91-95.

23. Tasca, T. and De-Carli, G. (2002): Shape variations of Trichomonas vaginalis in presence of different substrates. Parasitol. Latinoam., 57(1-2): 5-8.

24. Schwebke, R.; Venglarik, F. and Morgan, C. (1999): Delayed versus immediate bedside inoculation of culture media for diagnosis of 
vaginal trichomonosis. J. Clin. Microbiol., 37(7): 2369-2370.

25. Kaydos-Daniels, C. S. (2002): Improved detection of the urogenital pathogen Trichomonas vaginalis in men and women. M. D. thesis. Department of Epidemiology, School of Public Health, University of North Carolina, Chapel Hill.

26. Jatau, D. E.; Olonitola, S. O. and Olayinka, T. A. (2006): Prevalence of Trichomonas infection among women attending antenatal clinics in Zaria, Nigeria. Annal. African Medi., 5(4):178 - 181.
27. Abd El-Ail, A. A.; Habib, A. F. and El-Arousy, H. M. (2008): Assessing feasibility of urine samples for diagnosis of Trichomonas vaginitis by both wet mount and nested PCR. Internat. J. Medicine. 1(4): 154-156.

28. Malla, N.; Gupta, I. and Mahajan, C. R. (2001): Human trichomoniasis. Indian J. Med. Microbiol., 19(1):6-13.

29. Afzan,M.Y.;Sivanandan,S.;Kumar,G.S.(2010): Modified Field stain-a rapid stain for Trichomonas Vainalis. Diagnost. microbiol. and infect. Dis. (68) :159-162. 